Methods Case notes of all patients admitted with CAP over a 3 month period were requested and 175 were obtained. Information was gathered on the presence of underlying chronic lung conditions, CAP severity/mortality markers (SIRS and CURB65 scores) and mortality.

Results At least one underlying chronic pulmonary condition was found in $45.1 \%(\mathrm{n}=79)$, the commonest being COPD $(\mathrm{n}=56)$. CURB65 score was 0 to 1 in $39.4 \%$ (low risk), 2 in $27.4 \%$ (moderate risk), $3-5$ in $17.2 \%$ (high risk) and not done in $16 \%(\mathrm{n}=28)$. SIRS criteria were met in just under half of the cases $(48.5 \% \mathrm{n}=85)$.

An in-patient mortality review during this study period showed that $8 \%(n=14)$ CAP patients died in hospital within 30 days. An association of these patients with background lung condition, CURB65 and SIRS is shown in Table 1.

\begin{tabular}{|c|c|c|c|c|c|c|c|c|}
\hline \multirow[t]{2}{*}{ Mortality } & \multicolumn{2}{|c|}{ Chronic lung disease } & \multicolumn{2}{|c|}{ CURB65 } & \multicolumn{2}{|c|}{ SIRS } & \multicolumn{2}{|c|}{$\begin{array}{l}\text { SIRS and/or } \\
\text { CURB65 2-5 }\end{array}$} \\
\hline & Yes & No & $0-1$ & $2-5$ & Yes & No & Yes & No \\
\hline Numbers & 10 & 4 & 1 & 7 & 5 & 9 & 8 & 6 \\
\hline$\%$ & $12.6 \%$ & $4.2 \%$ & $1.4 \%$ & $9 \%$ & $5.9 \%$ & $10 \%$ & $9 \%$ & $7 \%$ \\
\hline
\end{tabular}

Conclusion We showed an improvement in mortality figures compared with the BTS National CAP adult audit 5 years ago ( $8 \%$ vs $18.3 \%$ ). A significant number of these patients have an underlying chronic lung disease which predisposed them to developing CAP. The highest mortality was seen in patients with a high CURB65 score with SIRS response.

\section{P211 IMPACT OF DOOR-TO-RADIOGRAPH TIME ON PNEUMONIA MANAGEMENT}

JM Dudziak, KL Scott, A Ashton, R Varia. Whiston Hospital, Prescot, Merseyside, UK

\subsection{6/thoraxjnl-2015-207770.347}

Introduction Pneumonia continues to be associated with significant mortality. ${ }^{1}$ The diagnostic gold standard remains the Chest X-Ray (CXR). Quality indicators such as door-to-antibiotic or door-to-radiograph time are monitored as surrogate outcome measures.

Objective To assess the impact of CXR availability at first clinician contact in cases where initial antibiotics were delayed.

Methods We interrogated the time of initial consultation, radiographs and diagnosis for 57 CAP patients between March 2013 and February 2014 whose initial antibiotics were delayed beyond $4 \mathrm{~h}$.

Results The median age was 77 (interquartile range 67-85), 32 $(53 \%)$ were female. Presentation was to the ED in $45(79 \%)$ and to the GP assessment unit (GPAU) in $12(21 \%)$ cases. 37 (65\%) cases had SIRS, $45 \%$ had a CURB-65 score of 3 or above.

CXR reports were compatible with pneumonia in 44 (77\%) cases, but only 11 (19\%) had a CXR at time of first doctor contact. Interestingly, a reported consolidation was not associated with an initial diagnosis of pneumonia ( $p=1.0000$, Fisher's exact test, two-tailed $\mathrm{p}$ ).
Median time to first clinician contact was 2:14 h (ED 2:03 h, GPAU 4:33 h). Overall, 20 patients $(35 \%)$ had a diagnosis of pneumonia after the initial consultation, 7 (58\%) in GPAU and $13(29 \%)$ in the ED. CXRs were obtained within $4 \mathrm{~h}$ in 49 $(86 \%)$ cases. Median time to diagnosis was 7:06 h (ED 8:35 h, GPAU 5:54 h). CXR availability at first clinician contact differed significantly - GPAU 50\%/6 vs ED 11\%/5 ( $\mathrm{p}=0.0068$, Fisher's exact test, two tailed p).

Discussion There were significant delays to diagnosis, despite most CXR reports indicative of pneumonia. The absence of a CXR on initial clinician contact may contribute to the poor diagnostic accuracy seen in this case series. Notably the $4 \mathrm{~h}$ door-toradiograph target set by BTS was largely met. We will deploy the GPAU pneumonia care bundle in the ED, which was shown to improve door-to-radiograph time (CURECAP, reported previously ${ }^{2}$ ). The efficacy of this intervention will be the subject of further studies.

\section{REFERENCES}

1 Gibson GJ, et al. Eur Respir J. 2013;42:559-563

2 Cunningham P, et al. Thorax 2013;68(Suppl 3):A186

\section{P212 MICROBIOLOGICAL SAMPLING IN COMMUNITY- ACQUIRED PNEUMONIA: DO WE FOLLOW THE GUIDELINES AND DOES IT HELP OUR PATIENTS?}

${ }^{1}$ SS Sunny, ${ }^{2} \mathrm{P}$ McDermott, ${ }^{2} \mathrm{~K}$ Tariq, ${ }^{2} \mathrm{~J}$ Hadcroft, ${ }^{1} \mathrm{~J}$ Folb. ${ }^{1}$ Medical Microbiology, Liverpool Clinical Laboratories, Royal Liverpool University Hospital, Liverpool, UK; ${ }^{2}$ Respiratory Medicine, Royal Liverpool University Hospital, Liverpool, UK

\subsection{6/thoraxjnl-2015-207770.348}

Introduction Community-acquired pneumonia (CAP) is a common cause for hospital admission and carries a high mortality rate. Choosing the correct antibiotic can be challenging and "atypical" pathogens, such as Mycoplasma pneumoniae and Chlamydophila pneumoniae, are not eliminated by some firstline empirical agents. Identification of the infecting organism through microbiological sampling can help to tailor antibiotic therapy and substantially improve patient outcomes. Guidelines exist (NICE, BTS, trust) that recommend which patients should undergo microbiological sampling. We wished to determine whether these guidelines were followed in our trust in patients admitted with CAP.

Methods We reviewed the notes of adult patients admitted over a 15 -week period (February-May 2014) with a clinical code of pneumonia. Further information was derived from hospital systems, including Telepath (used by the Microbiology department) and from 'Advancing Quality' data available for these patients.

Results 175 patients were identified with CAP. Blood cultures (BCs) were indicated in 89 patients according to trust guidelines (based on Systemic Inflammatory Response Syndrome (SIRS) criteria, CURB-65 score, immunocompromised; data sufficient in $147)$ and appropriately collected in 55 (61.8\%). However, only 4 had positive (clinically significant) BC results. Sputum samples were sent for 31 patients $(17.7 \%, \mathrm{n}=175)$ and only $19 \%$ had significant bacterial growth. All patients transferred to intensive care (ITU; 3.4\%) were screened appropriately for urinary pneumococcal antigen (UPA) and urinary legionella antigen (ULA), with 1 positive UPA result. 6 (of 10) UPA and 7 (of 12) ULA samples appeared to be sent inappropriately for non-ITU patients and were rejected by the laboratory. Serum samples were sent for Mycoplasma testing in 7 patients, despite this 
service being superseded by testing by polymerase chain reaction (PCR)-testing of nose/throat swabs, for which only 1 was sent for the entire cohort.

Conclusion While BC collection was frequent, compliance with local guidance was less than desired. Sampling to rule out atypical pathogens in CAP appeared to be low and, occasionally, inappropriate. Although positive detection rates were low, further work appears to be indicated to optimise patient outcomes in CAP by increasing awareness amongst clinicians of tests available and ensure appropriate sampling for atypical pathogen screening.

\section{P213 CXR FOLLOW-UP AFTER COMMUNITY ACQUIRED PNEUMONIA (CAP): OUTCOMES OF ADHERENCE TO GUIDELINES}

${ }^{1} \mathrm{P}$ Eaton, ${ }^{2} \mathrm{HJ}$ Curtis. ${ }^{1}$ Newcastle University, Newcastle Upon Tyne, UK; ${ }^{2}$ Queen Elizabeth Hospital, Gateshead, UK

\subsection{6/thoraxjnl-2015-207770.349}

Introduction and objectives BTS Community Acquired pneumonia (CAP) guidelines require a chest radiograph (CXR) 6 weeks after discharge in high risk patients. This is the hospital team's responsibility. Malignancy is reported in $1.7 \%$ of patients.

We wanted to assess how this was organised across our trust and the outcomes, aiming to improve organisation of the service.

Methods Patients were identified via retrospective review of local NIV/COPD and critical care ICNARC databases. Xrays and reports were reviewed and eRecords interrogated for appointments, follow-up CXR and outcomes.

Results 102 patients were identified between December 2013 to January 2015, (mean age 69 and 56 female patients). Only 54 patients had the follow up CXR. 16 patients did not need local follow-up for a variety of reasons: co-morbidities; current malignant diagnosis; CXR clear pre-discharge or usual residence outside geographical area. 7 patients did not attend the organised follow up appointment. Ultimately, 25 patients had no follow up plans made.

The majority of follow-up CXR were organised by the hospital team $(\mathrm{n}=53)$ compared to discharge correspondence instructions to GP to request a CXR $(\mathrm{n}=14)$. The hospital requested CXR were significantly more likely to be undertaken, with $88 \%$ completed versus $57 \%$ from requests to GP (p $<0.05$ Chi-square).

The majority of CXRs showed resolution of changes $(n=38$ / 54), 3 patients had lung or pleural malignancy diagnosed, 2 patients are having on-going nodule follow-up and 1 patient had atypical mycobacterium diagnosed. 10 patients to date have incomplete resolution of their changes. These end-points were after 62 CXRs, $7 \mathrm{Ct}$ scans, 2 bronchoscopies and 1 pleural biopsy.

Conclusion There is room for improvement within our trust to improve this parameter for CAP patients. This would be best fulfilled by automatic request at time of discharge follow CAP.

The follow up clearly requires increasing amounts of work and administration. How does the NHS keep up with guideline requirements and clinical outcomes in the ageing population?

The finding of $5.5 \%(3 / 54)$ new malignancy shows the importance of follow-up and is higher than published reports.

\section{P214 ARRHYTHMIAS IN PNEUMONIA: A REVIEW OF INCIDENCE, OUTCOMES AND MANAGEMENT}

DR Cox. University Hospital Aintree, Liverpool, UK

\subsection{6/thoraxjnl-2015-207770.350}

Background Community-acquired pneumonia (CAP) has high mortality, from 5 to $18.3 \% .^{1}$ Arrhythmias are a recognised significant complication. Growing evidence associates this treatable complication with increases in mortality. No review has summated data on mortality or ways to improve outcome.

Aims This review aimed to define the extent of the problem, collate and appraise evidence regarding outcomes and management, and identify gaps in understanding.

Methods Narrative review using a systematic protocol. Medline, ProQuest, Web of Science were searched for papers reporting adults with CAP complicated by arrhythmia. 382 articles were assessed and excluded based on title (348), abstract (27) and full text (6), leaving 11 . Review of bibliographies added 3, totalling 14. These were appraised and coded, with Newcastle-Ottawa scores assigned.

Results Three reviews and 11 primary studies were included: 10 Cohorts (4 prospective, 6 retrospective) and one case series. One meta-analysis of cardiac events identified a pooled incidence of $4.7 \%$ for CAP inpatients developing arrhythmia. ${ }^{2} \mathrm{~N}-\mathrm{O}$ scores ranged from 5 to 9, Median 7.5. Outcomes reported: Incidence; 30 and 90 day mortality; Re-hospitalisation; predisposing factors. Only one paper commented on treatment.

Discussion There is high quality evidence of a link between CAP and arrhythmogenesis. Data linking it to mortality suggest a strong association with worse outcome. This review was limited by its single reviewer. Some evidence was limited by retrospective study designs and biassed populations. The strengths of this review lie in its reproducible systematic methods and clear outlining of gaps in our understanding of this phenomenon, particularly regarding best management.

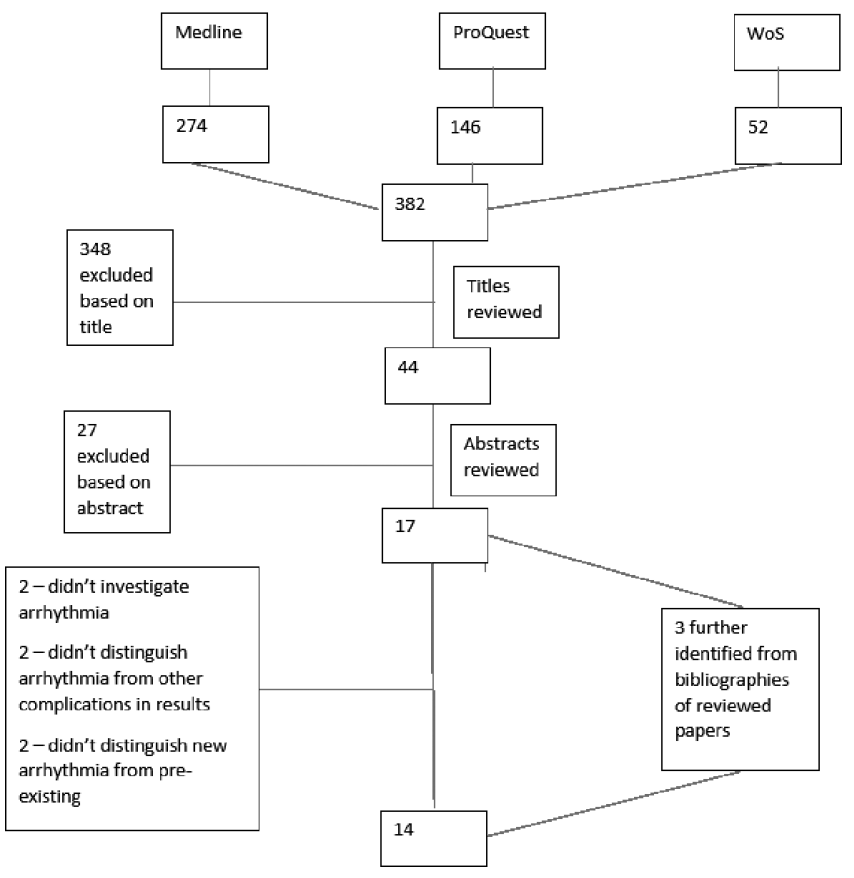

Abstract P214 Figure 1 